\title{
Entre intelligence collective et porosité augmentée, étude d'un médiateur entre chercheurs et praticiens
}

Between collective intelligence and added porosity, study of a mediator between researchers and practitioners

Laurent Morillon et Arlette Bouzon

\section{OpenEdition}

Journals

Édition électronique

URL : http://journals.openedition.org/communicationorganisation/4902

DOI : 10.4000/communicationorganisation.4902

ISSN : $1775-3546$

Éditeur

Presses universitaires de Bordeaux

Édition imprimée

Date de publication : 1 juin 2015

Pagination : 75-84

ISSN : 1168-5549

\section{Référence électronique}

Laurent Morillon et Arlette Bouzon, «Entre intelligence collective et porosité augmentée, étude d'un médiateur entre chercheurs et praticiens ", Communication et organisation [En ligne], 47 | 2015, mis en ligne le 01 juin 2018, consulté le 03 mai 2019. URL : http://journals.openedition.org/ communicationorganisation/4902 ; DOI : 10.4000/communicationorganisation.4902 


\title{
Entre intelligence collective et porosité augmentée, étude d'un médiateur entre chercheurs et praticiens
}

\author{
Laurent Morillon ${ }^{1}$ et Arlette Bouzon ${ }^{2}$
}

Dans une économie mondialisée à la concurrence accrue, l'innovation ${ }^{3}$ est présentée dans les discours étatiques français comme une clef majeure de compétitivité. Elle serait créatrice de valeurs et d'emplois en permettant de se différencier des pays à bas coût de main-d'œuvre et de demeurer en posture de donneur d'ordres. La recherche doit permettre cette innovation et servir la performance économique. Afin d'opérationnaliser et d'influencer la nature et les objectifs des projets menés, différents dispositifs sur appel d'offres font évoluer les modes de financement de la recherche publique : chaires industrielles, pôles de compétitivité, fondations... Les interactions entre chercheurs et praticiens sont en outre incitées par l'entremise de financements mixtes privés/publics. Pour les scientifiques ce phénomène ne va pas sans questionnements d'ordres éthique, épistémologique, théorique, pragmatique ou encore méthodologique. Dans le cadre du présent article, nous rendons compte de l'étude d'un dispositif médiateur particulier. Nous portons intérêt aux interactions constitutives d'un "laboratoire commun » associant un organisme de recherche publique et une entreprise, financé par l'Agence Nationale de la Recherche (ANR).

\footnotetext{
1 Laurent Morillon est maître de conférences en sciences de l'information et de la communication à l'Université de Toulouse, Paul Sabatier. Membre de l'équipe Organicom du LERASS (EA 827), ses recherches portent sur les interactions entre chercheurs et praticiens par l'entremise de leurs pratiques et modèles épistémologiques. Il codirige le laboratoire commun RiMec, financé par l'ANR, qui associe le laboratoire LLA-CRÉATIS et la PME Europa Organisation ; laurent.morillon@iut-tlse3.fr

2 Arlette Bouzon est professeur des universités en sciences de l'information et de la communication à l'Université de Toulouse, Paul Sabatier. Ses recherches au LERASS (EA 827) concernent l'activité humaine collective quand elle va au-delà d'une performance individuelle, quelle soit professionnelle ou, plus généralement, productrice de relations et d'interactions. Elles portent sur les équipes d'individus au travail et plus spécifiquement sur les situations de conception à plusieurs experts, qui offrent un champ privilégié d'étude de l'intelligence collective, permettant d'appréhender les liens existants entre communication et cognition, dans une perspective interactionniste élargie ; arlette.bouzon@iut-tlse3.fr

3 L'innovation " agrège au changement une finalité fonctionnelle et socio-économique contribuant à la création de valeur, et suppose une diffusion réussie vers ses utilisateurs au travers d'une transaction commerciale (Schumpeter, 1935)»(Bouzon, 2002 : 11).
} 
Dans une première partie sont évoqués les contextes qui incitent chercheurs et praticiens à interagir. Parmi les différents médiateurs identifiés, le laboratoire commun RiMeC est ensuite introduit. Enfin, certains des constats réalisés sur l'intelligence collective et la porosité à l'œuvre pour ce médiateur sont présentés.

\section{Des contextes qui incitent chercheurs et praticiens à interagir}

Pour le président de la République française, « la recherche sera un levier du redressementdela France $»^{4}$.L'Étatfrançais, dansla mouvance du rapport Berger de 2012, invite la recherche publique à investir les mondes socio-économiques et ceux-ci à contribuer à son financement. Crédits impôt recherche, laboratoires communs, chaires industrielles, Conventions Industrielles de Formation par la Recherche (CIFRE)... sont autant de dispositifs qui, pour certains depuis plus de trente ans, incitent à associer ressources publiques et privées. Ils entrent en outre en synergie avec des initiatives privées, telles par exemple le prix $\mathrm{AEF}^{5}$. Les occasions d'interactions entre praticiens et chercheurs se multiplient et les discours scientifiques, plus ou moins vulgarisés, tendent à davantage circuler dans les sphères professionnelles. Plus particulièrement en SIC, discipline par nature riche d'interfaces avec la société (Boure, 2002), le champ et les objets de la communication des organisations / organisationnelle induisent tout particulièrement des interactions avec les praticiens. La fréquentation des milieux professionnels est en effet courante pour ces chercheurs que ce soit pour produire des savoirs, décrire des phénomènes, ou encore, à l'invitation de praticiens, pour éclairer de manière distanciée les pratiques, apprécier des stratégies et/ou proposer des connaissances opérationnalisables (Morillon, Bouzon, Lee, 2013). Qu'il s'agisse d'une recherche, d'une étude voire d'une intervention, " la demande d'un savoir centré et spécifié aux besoins des professionnels existe et grandit " (Meyer, 2006 : 108). Les deux catégories d'acteurs trouvent intérêt à collaborer : les praticiens se distancient de leur pratique quotidienne et/ou, dans une optique performative, espèrent transformer théories et analyses de chercheurs en connaissances opérationnelles; les chercheurs peuvent valoriser leurs travaux, recenser des expériences de praticiens, initier de nouveaux terrains (pour observer ou mettre à l'épreuve des théories), et parfois des commandes, la dimension économique de la valorisation devenant alors première.

\footnotetext{
4 Discours de François Hollande, du 4 février 2013 au collège de France, www.enseignementsup-recherche. gouv.fr/cid67060/discours-de-francois-hollande-au-college-de-france.html - dernière consultation le 6 mars 2015 .

5 www.rue-aef.com/prix-aef/ - dernière consultation le 15 avril 2015.
} 
Que ce soit à l'initiative du monde académique ou socio-économique, praticiens et chercheurs se rencontrent, collaborent, parfois se confrontent par l'entremise de certains médiateurs que nous nous proposons à présent d'aborder.

\section{Questionner les médiateurs, le cas du Labcom RiMeC}

Il existe une diversité de médiateurs mettant en relations chercheurs et praticiens. Il peut s'agir de documents (revue et ouvrage, presse spécialisée, manuel...), d'événements (colloque, journée d'étude, conférence, rencontre professionnelle...), d'organisations dédiées (par exemple l'ANVIE $^{6}$ ) ou encore de dispositifs, qu'ils soient de formation (initiale ou continue dans les universités) ou de recherche (laboratoire commun, CIFRE, recherches impliquées et actions...). Ces médiateurs favorisent les échanges, les confrontations voire les constructions collectives entre chercheurs et praticiens. Ils peuvent également susciter des réflexions dans la mise en rapport dialogique de l'épistémè et de la praxis ${ }^{8}$. Si celle-ci peut sembler relativement naturalisée pour les disciplines des sciences exactes, pour certaines SHS elle demeure plus récente et potentiellement polémique. Elle peut mettre en exergue certains stéréotypes, incompatibilités temporelle et de projet, risques pour la science, voire potentielles déviances sociétales, les arguments idéologiques devenant prégnants. Différents médiateurs ont dès lors été étudiés : CIFRE (Morillon, Gardère, 2010), manuels de communication des organisations (Morillon, Gramaccia, 2013), journées professionnelles (Morillon, 2013), organisations (Morillon, Martinez, 2013) et recherches actions (Bouzon, Meyer, 2008). Dans le cadre du présent article, nous proposons une étude scientifique d'un «Labcom».

Depuis 2013, l'ANR soutient le dispositif des laboratoires communs, dits «Labcom». Il incite les acteurs de la recherche publique à créer des partenariats structurés avec de Petites et Moyennes Entreprises (PME) ou des Entreprises de Taille Intermédiaire (ETI) : « la création commune de connaissances ou de savoir-faire entre les organismes de recherche et ce type d'entreprise peut être un facteur important d'innovation, de compétitivité des entreprises, et donc de création d'emplois. La constitution de liens bilatéraux, s'inscrivant dans la durée, entre les PME/ETI et les organismes de recherche constitue un enjeu important dans la chaîne de l'innovation $»^{9}$. Le laboratoire doit disposer d'une gouvernance commune, d'une feuille de route de recherche et d'innovation, de moyens ainsi que d'une stratégie de valorisation du travail partenarial.

6 L'ANVIE née en 1991 se veut un organisme de médiation entre la recherche en sciences humaines et sociales et les entreprises - www.anvie.fr - dernière consultation le 8 mars 2015.

7 Soit la démarche de mise en abstraction intellectuelle des connaissances et de leurs présupposés.

8 Soit la mise en œuvre dans le monde physique d'idées dont le développement peut faire l'objet de réflexivité.

9 www.agence-nationale-recherche.fr/fileadmin/aap/2015/aap-labcom-2015.pdf - dernière consultation le 5 mars 2015. 
À la suite d'un appel à projets, l'ANR finance ceux retenus à hauteur de 300000 euros sur trois ans pour les activités de la phase de montage et de fonctionnement.

En 2010, des chercheurs et acteurs du monde socio-économique toulousain ont créé un dispositif atypique de production scientifique favorisant les collaborations entre universitaires des SHS, artistes et organisations ${ }^{10}$. C'est dans cette dynamique que le Labcom RiMeC (Réinventer le Média Congrès) est né. Il associe le laboratoire LLA-CRÉATIS de l'Université Toulouse JeanJaurès et une PME, Europa Organisation, leader national dans l'organisation de congrès médicaux. Ce Labcom doit servir les intérêts des chercheurs et des praticiens. Ces derniers attendent notamment un avantage concurrentiel en proposant à terme des sessions innovantes dans leurs congrès. Les chercheurs souhaitent produire des savoirs inédits dans différentes disciplines (SIC, éducation, art). En générant une recherche " en acte " rendant " interappartenant " processus de recherche et processus observés, ils espèrent accéder à certains phénomènes de la réalité sociale. Le projet scientifique est de penser les formes de communication dans les congrès, considérés comme des espaces de formation et de socialisation. Le congrès est appréhendé comme un média à déconstruire afin d'en réagencer les éléments dans une perspective intermédiatique. Le concept d'intermédialité doit permettre d'éclairer les relations, interactions, imbrications, enchaînements et interdépendances en contexte entre médias. La démarche scientifique de prospective (innovation de rupture versus innovation incrémentale), en s'inscrivant dans le temps long, en n'appliquant pas de modèles pré-construits et en produisant avant tout des savoirs, se démarque de celle de la consultance.

L'un des auteurs de cet article codirige ce Labcom dont la dynamique scientifique donne à réfléchir sur les enjeux et les conditions de mise en œuvre des recherches menées. RiMeC apparaît en effet comme un espace privilégié pour appréhender certaines des relations entre praticiens et chercheurs, entre pratiques professionnelles et de recherche et de manière générique entre théorie et pratique. De telles réflexions s'inscrivent en SIC dans la lignée des travaux sur les praticiens de la communication et leurs modèles (cf. par exemple Fourrier, Martin-Juchat, Lépine, 2010) et sur ceux, plus rares, qui s'intéressent aux liens entre chercheurs et praticiens (par exemple Couzinet, 2003 ; Bouzon, Meyer, 2008). Ne participant pas aux travaux menés sur le terrain, afin de repérer les interactions constitutives, nous adoptons une démarche compréhensive et initions une observation participante longitudinale avec un recueil des documents diffusés lors des différentes réunions de coordination scientifique et de présentation des résultats. L'étude d'une seule organisation ne nous permet pas de généraliser nos constats. Ce travail nous donne toutefois l'occasion de réfléchir plus avant quant à la réalité des logiques en

10 Association STARS et plateforme de recherche CRISO - http://artetscience.eu/recherche/ - dernière consultation le 13 avril 2015. 
présence dans cette étude de cas. Il ne s'agit pas en outre de discuter les enjeux éthiques ou déontologiques du dispositif, de réfléchir aux statuts respectifs de la science et du chercheur face aux attentes sociales, ni de questionner le statut et la légitimité des connaissances issues de recherches appliquées. Nous nous proposons plus modestement de discuter deux phénomènes dans le cadre restreint de cet article.

\section{RiMeC, espace d'intelligences collectives et médiateur à porosité augmentée ?}

L'étude des interactions constitutives du Labcom nous permet de le caractériser comme espace d'intelligences collectives et comme médiateur à "porosité augmentée ».

\section{Un espace de déploiements d'intelligences collectives}

Lintelligence collective (IC), en tant que concept à la mode, dispose de diverses définitions selon le contexte, l'usage voire, dans une perspective scientifique, la discipline considérée. Si elle est parfois associée aux sociétés d'insectes, dans une optique performative que nous ne retiendrons pas, elle peut être entendue comme une hypothèse relative à la capacité d'un groupe à atteindre, dans l'action et collectivement, une performance d'un niveau supérieur (Belin, 2006). Nous privilégierons ici une définition où l'IC est considérée comme une propriété émergente du collectif dont le fonctionnement dépend du réseau de connexions entre acteurs pour construire collectivement un sens à la situation (Bouzon, 2004).

$\mathrm{Au}$ sein du Labcom, il est possible de distinguer deux espaces de déploiement potentiel d'IC : l'un pour le collectif de chercheurs, l'autre pour celui des chercheurs et des praticiens ${ }^{11}$. Pour les six chercheurs impliqués (un professeur, un maître de conférences, deux post-doctorants, un docteur et un doctorant), la construction collective du sens de la situation a d'abord été menée pour certains d'entre eux lors de la rédaction du projet soumis à l'ANR. La diversité des disciplines convoquées pour penser la complexité (SIC, sciences de l'éducation, sciences du langage, espagnol) et le rapport de celles-ci à la recherche applicative ont nécessité plusieurs concertations et ont induit certains ajustements et compromis. Suite à l'obtention du financement, la construction débute et se poursuit aujourd'hui encore, notamment lors de réunions de travail au minimum mensuelles. Celles-ci sont découpées en trois temps : l'équipe opérationnelle rend compte à la direction scientifique des avancées; des décisions collectives sont prises sur les objectifs, les modes opératoires, les rendus dans le respect du calendrier initialement fixé ; enfin, l'un des membres de l'équipe présente un travail de recherche susceptible d'intéresser le projet et/ou de sensibiliser les autres chercheurs à un concept particulier. De manière méta, ces séances participent

11 Notre mode d'investigation ne nous a pas permis d'accéder à l'espace des praticiens seuls. 
de la définition de nouvelles pratiques de production de savoirs rigoureux en repensant notamment certaines articulations entre recherches fondamentale et applicative.

Le collectif constitué par les chercheurs et les praticiens est moins institué mais il semble être un second espace de déploiement potentiel d'IC. En effet, si le projet commun initié est d'abord né d'une volonté de rencontre entre praticiens de la recherche et de l'action, il ne s'inscrit toutefois pas dans une relation classique et asymétrique de chercheurs "prestataires » et d'organisations « commanditaires » ayant pour objet de produire une recherche exclusivement appliquée. Ainsi, la collaboration a débuté par la construction progressive d'une définition partagée de la situation et la rédaction du projet soumis à l'ANR. L'ancrage social de la recherche a été négocié afin de prioriser l'échange intellectuel et éviter une relation client-fournisseur asservissante. Les obligations de réciprocité et d'équilibre permettent au chercheur de fournir un service de recherche et de développement en échange de terrains, de moyens et d'une relative autonomie expérimentale. Une fois le projet lancé, l'entrée sur le terrain s'est réalisée progressivement. Par exemple pour le projet d'innovation dans les sessions, les chercheurs ont d'abord mené des observations non participantes sur des congrès et des réunions préparatoires de praticiens. Ils ont ensuite participé de manière plus active à ces réunions avant de proposer des expérimentations à différentes équipes de direction (du congrès, de l'entreprise, de la société savante commanditaire) et opérationnelles. Ils ont enfin travaillé avec les médecins qui sont amenés à animer ces sessions innovantes. Ces différentes réunions (préparatoires, de travail, de restitution) donnent lieu à des interactions entre des acteurs très différents (par exemple des chefs de projets pragmatiques avec des chercheurs issus de disciplines " classiques ", parfois abstraites) qui ne se seraient pas rencontrés sans le Labcom. Les participants échangent autour de problématiques communes et ajustent leurs conceptions : « le travail collectif ainsi partagé devient une source d'avancée pour chaque interlocuteur pris individuellement »(Albero, Robin, 2004 : en ligne). La dialectique entre savoirs théoriques et pragmatiques permet in fine à la recherche de devenir utile à l'action autant que l'implication du chercheur est utile à la recherche.

\section{Un médiateur à porosité augmentée}

Si les « mondes » scientifiques et socio-économiques tendent à se rapprocher et parfois à se confondre, leurs activités, visées, modèles, concepts, vocabulaires, référents et indicateurs respectifs créent une frontière permettant de les distinguer. En ouvrant un espace d'interactions entre chercheurs et praticiens, les médiateurs précédemment évoqués peuvent rendre poreuse cette frontière. La porosité sera donc ici considérée comme la capacité d'un médiateur à rendre perméables des mondes différents. Il est possible de distinguer différents degrés de porosité : un document dont la lecture donne lieu à une construction 
individuelle du sens en contexte n'aura pas la même porosité qu'un événement qui autorise un contact direct et peut induire certaines interactions ou qu'une collaboration qui oblige ne serait-ce qu'à des interactions de coordination. $\mathrm{Au}$-delà de la modification de représentations respectives, nous pouvons alors considérer que cette porosité est « augmentée » lorsque les interactions induisent certaines hybridations (conceptuelles, épistémologiques, étymologiques, référentielles...) comme celles que nous avons déjà observées lors de l'étude d'une journée professionnelle (Morillon, 2013).

Le Labcom RiMeC met en relations trois années durant des chercheurs et des praticiens. Ainsi, l'équipe opérationnelle fréquente-t-elle le dirigeant, les chefs de projet ou encore des médecins conférenciers. Elle participe à différents congrès afin de mieux connaître les spécificités de l'organisation, ses contextes et ses produits. Les praticiens participent pour leur part aux investigations qui permettent d'élaborer et d'expérimenter "in vivo " de nouveaux formats de sessions dans les congrès médicaux. Et si les praticiens ont acculturé les chercheurs à leurs savoirs et savoir-faire, ceux-ci ont produit certains médiateurs de connaissances : documents de synthèse, réunions, fiches de traduction. Le Labcom semble donc offrir un contexte favorable à une porosité augmentée. Cette organisation n'existant que depuis une année, nous ne pouvons aujourd'hui circonscrire les hybridations abouties. Nous choisissons de réfléchir ici à celles concernant l'étymologie.

La collaboration suppose un langage et un système de représentations partagés, préalablement acquis ou co-construits. Ainsi l'équipe opérationnelle s'est-elle approprié certains des vocabulaires spécifiques à l'organisation (par exemple " facilitateur ", " topic », " core team ») ou au monde médical (" radiale », « french »...). En parallèle, lors des réunions de travail ou de restitution, un vocabulaire scientifique est présenté aux praticiens. Cette démarche peut déboucher sur une appropriation de concepts scientifiques permettant l'action (tel est le cas par exemple pour « média » ou « dispositif »). Mais au-delà de cette appropriation et des questionnements scientifiques induits, qu'en est-il de potentielles hybridations ? Un lexique commun est en cours d'élaboration dont l'objectif est de co-construire des définitions partagées pour un vocabulaire donné. Une définition courte pour treize termes ${ }^{12}$ identifiés comme utiles à l'activité du Labcom a été ainsi demandée à chacun de ses acteurs. Le but n'est pas de produire une définition objective mais d'établir la valeur d'usage de ces termes, soit leur signification «située » vis-à-vis des pratiques professionnelles respectives. L'un des post-doctorants a ensuite proposé un document de synthèse devant servir de support à une réunion de travail entre chercheurs et praticiens. Celle-ci peut donner l'occasion aux chercheurs de "traduire », au sens de Akrich, Callon et Latour (2006), certaines des définitions scientifiques susceptibles de faire évoluer

12 Artiste, communication, dispositif, expert, information, interactivité, formation, média, participant, performance, réception, spectateur et transmission. 
celles des praticiens et également donner lieu à des co-constructions de définitions pour un vocabulaire scientifique et technique spécialisé à l'aire d'emploi restreinte (Phal, 1969).

\section{Conclusion}

Malgré la difficile interpénétration des univers de recherche et d'action, notamment pour certaines SHS, le Labcom apparaît comme un médiateur à porosité augmentée. Afin de continuer à le caractériser, il pourrait être intéressant de l'appréhender comme espace de rencontres et comme agent. Il pourrait en effet être considéré comme un espace de rencontres matériel (lieu et temps), culturel (cadres de référence, conceptions, langages) ou encore déontologique (symétrie des relations et respect des spécificités propres à chaque métier), (Albero, Robin, 2004). Il pourrait également être appréhendé comme un agent : il circonscrit les activités de recherche et de terrain; permet l'organisation de l'activité, la confrontation d'expériences, l'émergence, la découverte et la rencontre (Bernard, 2006) ; fait échanger des acteurs aux statuts et projets différents sur une thématique commune. Il disposerait donc d'une capacité d'agir (Cooren, Taylor, Van Every, 2006).

$\mathrm{Au}$ final, si de nouvelles manières de penser la communication peuvent permettre d'améliorer la pratique, les savoirs produits sont susceptibles de faire avancer la science. Mais la qualité des travaux des chercheurs, a fortiori lorsqu'ils sont impliqués, ne peut être garantie sans une distance critique visà-vis des phénomènes interactionnels entre chercheurs et praticiens. C'est l'un des objets de cet article.

\section{BIBLIOGRAPHIE}

ALBERO B., ROBIN J.-Y., " Entre chercheurs et praticiens : traits d'union ou démarcation ? », Recherches \& éducations, $\mathrm{n}^{\circ}$ 8, 2004, en ligne :

http://rechercheseducations.revues.org/index $342 . h t m l$

AKRICH M., CALLON M., LATOUR B., Sociologie de la traduction - textes fondateurs, Paris, Les presses de l'école de Mines de Paris, 2006.

BELIN O., Intelligence collective et complexité : L'appropriation d'une technologie d'intelligence collective: Approches systémiques et affordancielles, thèse de doctorat en sciences de l'information et de la communication, Université de Montpellier 3, 2006.

BERNARD F., « Le laboratoire des sciences de l'information et de la communication. Entre prise, emprise et déprise des pratiques en information et communication ", $\mathrm{XV}^{\mathrm{e}}$ congrès de la SFSIC, Questionner les pratiques d'information et de communication. Agir professionnel et agir social, Bordeaux, mai 2006, p. 1-17.

BOURE R., Les origines des sciences de l'information et de la communication: regards croisés, Paris, Presses universitaires du Septentrion, 2002, p. 153-182. 
BOUZON A., La place de la communication dans la conception de systèmes à risques, L'Harmattan, Paris, 2004.

BOUZON A., MEYER V. (dir.), La recherche action en communication organisationnelle, L'Harmattan, Collection communication et civilisation, Paris, 2008

COOREN F., TAYLOR J. R., VAN EVERY E. J., Communication as organizing: Empirical explorations into the dynamic of text and conversation. Mahwah, NJ, Lawrence Erlbaum, 2006.

COUZINET V., " Praticiens de l'information et chercheurs : parcours, terrains et étayages ", Documentaliste, sciences de l'information, 40(2), 2003, p. 118-125.

FOURRIER C., MARTIN-JUCHAT F., LEPINE V., "Y a-t-il un modèle de communication dominant dans les organisations ou une hybridation dans les pratiques des communicants ? ", XVII" congrès de la SFSIC, Au cœur et aux lisières des SIC, Dijon, 2010, p. 437-443.

MEYER V., "De l'utilité des recherches-actions en SIC ", Communication Eซ organisation, $\mathrm{n}^{\circ} 30,2006$, p. 89-108.

MORILLON L., "Praticiens et chercheurs en communication des organisations : une pluralité d'hybridations lors d'événements médiateurs ", Colloque international Formes et enjeux contemporains de la communication et de la culture scientifiques et techniques, Grenoble, 27-29 novembre 2013.

MORILLON L., BOUZON A., LEE C., «Épistémologies, théories et pratiques professionnelles en communication des organisations », Études de communication, ${ }^{\circ} 40$, 2013, p. 9-23.

MORILLON L., GARDERE E., « Le doctorant CIFRE en communication des organisations : un jeune chercheur entre implication et distanciation », Loneux C., Parent B. (dir.), Communication des organisations, recherches récentes, tome 2, L'Harmattan, 2010, p. $185-196$.

MORILLON L., GRAMACCIA G., " Pour une approche critique des modèles dans les manuels de communication des organisations », Huet R., Vidaillet B., Heller T. (dir.), Communications-organisations et pensées critiques, Presses universitaires de Lille Septentrion, collection « capitalisme, éthique et institution », 2013, p. 363-373.

MORILLON L., MARTINEZ M., "Le chercheur, un auteur entre spectateur et acteur ", Colloque Acteurs, auteurs, spectateurs? Quelle place et quel(s) rôle(s) pour les individus et les groupes au sein des dispositifs et des processus communicationnels? 21-22 novembre 2012, Dijon, p. 313-323. En ligne : http://cimeos.u-bourgogne.fr/2014-0523-08-29-43/colloque-auteurs-acteurs-spectateurs.html

PHALA., «La recherche au CREDIF : la part du lexique commun dans les vocabulaires scientifiques et techniques », Le lexique, Langue Française, n ${ }^{2}$, Larousse, Paris, 1969

SCHUMPETER J. A., Théorie de l'évolution économique, Dalloz, Paris, 1935.

Résumé : L'innovation est présentée dans les discours étatiques français comme une clef majeure de compétitivité. Les interactions entre chercheurs et praticiens sont incitées notamment par des financements mixtes privé/public. Mais une telle tendance ne va 
pas sans questionnements pour les chercheurs. Dans le cadre du présent article, nous proposons l'étude d'un laboratoire commun qui associe une PME et un laboratoire public pour contribuer au renouvellement des formes de communication dans les congrès médicaux. L'observation de certaines des interactions constitutives de ce dispositif fait apparaître un médiateur à porosité augmentée, espace de déploiement d'intelligences collectives.

Mots clefs : chercheur, praticien, médiateur, intelligence collective.

Abstract : Innovation in the speeches given by the French government is presented as a major resource for competitiveness. Interactions between researchers and practitioners are encouraged by private/public funding. But, for researchers several questions about this trend remain unanswered. We propose here to study a laboratoire commun, a structure associating a mediumsized company and a public laboratory for renew communication in medical congresses. The study of some of constituent interactions reveals a porous mediator, area of collective intelligence. Keywords : researcher, practitioner, mediator, collective intelligence. 